1998-12-01

\title{
Measurement of Degree of Order in Mixed Polarised Fluorescent Polymer Liquid Crystal Films
}

\author{
Andrew Davey \\ Trinity College Dublin \\ Robert Howard \\ Technological University Dublin, Robert.Howard@tudublin.ie \\ Bernd Lahr \\ F.H. Darmstadt
}

See next page for additional authors

Follow this and additional works at: https://arrow.tudublin.ie/nanolart

\section{Recommended Citation}

Davey, A. et al. (1998) Measurement of degree of order in mixed polarised fluorescent polymer liquid crystal films. Molecular Crystals and Liquid Crystals, Vol. 325, pp. 79 - 90. December. doi.org/10.21427/ csns-2q35

This Article is brought to you for free and open access by the NanoLab at ARROW@TU Dublin. It has been accepted for inclusion in Articles by an authorized administrator of ARROW@TU Dublin. For more information, please contact arrow.admin@tudublin.ie, aisling.coyne@tudublin.ie,gerard.connolly@tudublin.ie.

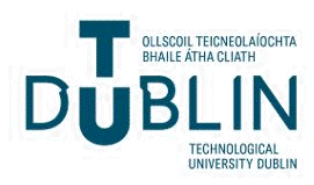




\section{Authors}

Andrew Davey, Robert Howard, Bernd Lahr, and Hugh Byrne

This article is available at ARROW@TU Dublin: https://arrow.tudublin.ie/nanolart/16 


\title{
Measurement of Degree of Order in Mixed Polarised Fluorescent Polymer Liquid Crystal Films.
}

Andrew P. Davey,* Robert G. Howard, Bernd Lahr ${ }^{(a)}$ and Werner J. Blau.

Department of Physics, University of Dublin, Trinity College, Dublin 2, Republic of Ireland.

(a) Visiting student from F. H. Darmstadt, Germany.

Hugh J. Byrne.

School of Physics, Dublin Institute of Technology, Kevin Street, Dublin 2, Republic of Ireland.

\begin{abstract}
Raman spectroscopy is used to investigate the degree of order found in in situ photopolymerised films exhibiting polarised emission. Studies indicate that the mixed system shows differing degrees of order for its two molecular components. The degree of order was measured in the liquid mesophase for the mixed monomers prior to polymerisation and was found to be in close agreement with the order of the two components measured in equivalent cured films.
\end{abstract}

\section{Introduction}

The last few years has seen many advances in the field of organic optoelectronics, specifically, light emitting devices using polymers and other smaller molecules. ${ }^{1}$ The performance of such devices has improved considerably due to optimisation of material interfaces, improvement of quantum yield and a better understanding of charge transport and degradation processes in amorphous organic systems.

The point has now been reached where more sophisticated devices such as organic microcavity structures ${ }^{2}$ and polarised emitters ${ }^{3}$ are beginning to be reported. Fabrication of polarised emitting films has employed a number of different alignment techniques. ${ }^{4-6}$ These techniques have been applied with varying degrees of success; there are still some problems to overcome. All of the techniques have so far failed to produce particularly high degrees of linear polarisation.

The approach which we have used in an attempt to overcome some of these problems is in-situ photopolymerisation of functionalised oriented liquid crystals to produce highly aligned chromophore networks. This technique has been studied for a number of years now ${ }^{7}$ for potential application in solid state liquid crystal display devices. Its exploitation in other areas is still however limited.

To produce these oriented polymer networks, in-situ photopolymerisation of macroscopically oriented mixtures of liquid crystal (LC) diacrylates was used. . Figure 1 depicts the photopolymerisation process in the liquid crystal phase. This 
technique involves the macroscopic alignment of the LC diacrylates and the 'freezingin' of the orientation by photo-polymerisation. Previous work has invariably employed molecules where the saturated carbon chains are positioned at either end of the long axis of the liquid crystal core. ${ }^{7}$ In our case however, the alkyl chains are bonded across the short axis of the core (see Figure 1). Networks obtained by this method are highly cross-linked and well ordered. This high degree of orientation is both thermally and temporally stable.

Polarisation dependent absorption and fluorescence has already been studied in these films ${ }^{8}$ and not unusually, absorption and fluorescence measurements imply different degrees of order. To study the degree of order on the molecular scale, we undertook the polarisation dependent Raman studies described herein in an attempt to obtain a better understanding of the organisation on a molecular scale.

\section{Results and Discussion}

In this study, we sought to utilise the long range ordering characteristics of liquid crystals to produce a thin film which would preferentially emit light in a particular polarisation and would exhibit good thermal stability properties. We therefore set out to design and synthesise a liquid crystal with an emissive functional core and crosslinkable side-groups. Our aim was to utilise such a liquid crystal in an ordered thin film form.

\section{Phase Transition Studies}

In order to produce oriented films with linearly polarised emission characteristics, a chromophore with a blue/violet emissive core and acrylate functionalities (FLLC) was synthesised (Figure 2). ${ }^{8}$

The phase transitions of FLLC were studied using differential scanning calorimetry (DSC) and polarising microscopy. The material is crystalline at room temperature and on heating shows a phase transition at $38^{\circ} \mathrm{C}$ to an unidenified intermediate phase. The transition from this intermediate phase to the isotropic phase has an onset temperature of $67^{\circ} \mathrm{C}$ and peak is at $80^{\circ} \mathrm{C}$. The sample exhibits no phase transitions when it is cooled from the isotropic phase until below $30^{\circ} \mathrm{C}$ where it crystallises.

\section{Long range ordering}

A mixture of FLLC, 2\% mol. of photoinitiator (CIBA-Irgacure ${ }^{\mathrm{TM}}$ 651) and 0.1 mole percent of thermal inhibitor (4-methoxyphenol) was prepared.

A glass cell was filled with the mixture in molten form by capillary action. The sample was cooled overnight to allow recrystallisation. The sample was heated to its intermediate state $\left(50^{\circ} \mathrm{C}\right)$ and any ordering in the film was observed through cross- 
polarisers under a microscope. Unfortunately no long range ordering was observed in this intermediate phase.

In order to produce a low viscosity nematic phase, 5\% by weight of FLLC was mixed with 1,4-phenylene bis\{4-[6-(acryloyl-oxy)hexyloxy]benzoate $(\mathbf{C 6 H})$, a previously studied cross-linkable nematic liquid crystal. ${ }^{9}$ The same proportion of photoinitiator and thermal inhibitor as before was then added. This new mixture exhibits a crystalline-nematic phase transition at $116^{\circ} \mathrm{C}$ and a nematic to isotropic transition at $150^{\circ} \mathrm{C}$. Glass cells containing this mixture were found to exhibit long range in the nematic phase. It is possible to 'freeze-in' this long range ordering by photopolymerisation. This procedure was found to be effective for loadings of up to 15\% by weight of FLLC.

Films prepared in this manner were previously studied for optical absorption and emission anisotropy. ${ }^{8}$ It was found that the two different experiments implied different degrees of order. We have therefore employed Raman spectroscopy to determine the nature of order on the molecular scale in these films.

\section{Raman Anisotropy Studies}

In an attempt to gain greater insight into the degree and nature of ordering in these systems, we undertook Raman inisotropy studies.

Raman spectroscopy was chosen specifically because it was found that the carboncarbon triple bond stretch from FLLC was too weak to be observed in the IR spectrum of cured films. The thin film form of the samples makes them much easier to measure using Raman spectroscopy than infrared. It was also found that the Raman spectra contains far fewer peaks and it is therefore simpler to interpret spectra and measure absolute peak intensities.

The studies were designed to probe the chemical nature of ordering in these systems. This was achieved by measuring the polarisation dependence of Raman peaks which may be assigned discreetly to modes exhibited by either the C6H or the FLLC moiety in the mixed, photopolymerised films.

In order to study order in the films, pump polarisation dependent Raman spectra were recorded. To distinguish between modes associated with the host and fluorescent guest, the Raman spectra of the two monomers in powder form were first recorded (Figures 3a and 3b).

The spectral region of interest here is from 1585 to $2250 \mathrm{~cm}^{-1}$ since here there are peaks which may be unambiguously assigned to either C6H or FLLC molecular 
cores. For FLLC, the peaks at 1590 and $2200 \mathrm{~cm}^{-1}$ are of interest. These are assigned to a phenyl mode and a carbon-carbon triple bond mode respectively. For $\mathbf{C 6 H}$, the peaks at $1600 \mathrm{~cm}^{-1}$ and $1730 \mathrm{~cm}^{-1}$ are assigned as phenyl ring and carbonyl modes respectively.

It is interesting to note the absence of a peak at around $1730 \mathrm{~cm}^{-1}$ in the spectrum of FLLC (which also contains carbonyl bonds in the acrylate functionalities). The presence of this peak in the spectrum of $\mathbf{C 6 H}$ is therefore probably from the ester linkages and not from the acrylate end groups.

Equivalent peaks to these at practically identical frequencies were found in cured film mixtures and thus it is assumed that the in situ photopolymerisation process has no effect on the positioning of these peaks. Initial investigations showed that all four peaks shared a similar polarisation dependent intensity variation.

The angular dependence of Raman scattering intensity was therefore investigated for these four peaks in a 10\% FLLC; 90\% $\mathbf{C 6 H}$ in situ cured film. Raman spectra were recorded for various angles with respect to the orientation direction and the integrated intensity dependence for the four peaks is shown in Figures 7a and 7b. Figure 4a shows the angular dependence of intensity for the two peaks associated with FLLC and Figure 4b shows those for $\mathbf{C 6 H}$.

As expected, all of the peaks show a symmetrical, periodic variation of intensity with angle. This demonstrates ordering of both the guest and host in these films.

In order to compare the degree of order of C6H and FLLC in the films, the order parameter (S) was determined from the measurements. $S$ is defined here as:

$$
\mathrm{S}=\frac{\mathrm{I}_{\mathrm{pa}}-\mathrm{I}_{\mathrm{pe}}}{\mathrm{I}_{\mathrm{pa}}+2 \mathrm{I}_{\mathrm{pe}}}
$$

where $I_{p a}$ and $I_{p e}$ are respectively the peak intensities parallel and perpendicular to the orientatation direction.

In order to determine values of $\mathrm{I}_{\mathrm{pa}}$ and $\mathrm{I}_{\mathrm{pe}}$ for the four peaks, the data shown in Figure 2 was fitted to the following function:

$$
\mathrm{I}(\theta)=\mathrm{A} \cos ^{2}(\theta-\mathrm{B})+\mathrm{C}
$$

where A is an amplitude term, B is a phase correction accounting for any discrepancy between 'internal' and 'external' angle and C accounts for finite intensity at minima. Equation 1 may now be rewritten as: 


$$
S=\frac{A}{A+3 C}
$$

Values of S for the four peaks were then determined from the fits (shown in Figure 4) and are listed in Table 1.

The values obtained are clearly consistent within errors. These results suggest a distinct difference in the degree of order of $\mathbf{C 6 H}$ and FLLC in the photopolymerised film. C6H shows a far higher degree of ordering than FLLC perhaps due to the fact that the flexible side chains in FLLC are positioned either side of the rigid core whereas in $\mathbf{C 6 H}$, they are at either end of the long axis. This may limit the degree of ordering FLLC can achieve in the mesophase and consequently in the photopolymerised film.

It has been shown previously ${ }^{9}$ that the temperature of photopolymerisation affects the order in $\mathrm{C6H}$ films. The polymerisation temperature is usually expressed in terms of 'reduced' temperature $\left(\mathrm{T}_{\mathrm{r}}\right)$ :

$$
\mathrm{T}_{\mathrm{r}}=\frac{\mathrm{T}_{\mathrm{pol}}}{\mathrm{T}_{\mathrm{cl}}}
$$

Where $T_{p o l}$ is the polymerisation temperature and $T_{c l}$ is the clearing temperature (or temperature at which transition to the isotropic phase occurs). To investigate the dependence of order on reduced temperature, Raman studies similar to those already outlined were carried out on 5\% FLLC cured films with $\mathrm{T}_{\mathrm{r}}$ values of 0.7, 0.8 and 0.9. For convenience, only two peaks were considered; $1730 \mathrm{~cm}^{-1}(\mathbf{C 6 H})$ and $2210 \mathrm{~cm}^{-1}$ (FLLC) The findings of these studies are summarised in Table 2.

As might be expected, there appears to be a downward trend in the order of both $\mathbf{C 6 H}$ and FLLC as $T_{r}$ increases (and the isotropic phase is approached). Again, it is clear that the FLLC is not as highly ordered as the $\mathbf{C 6 H}$.

These results indicate possible reasons for the discrepancy in the previous absorption and photoluminescence measurements. Since FLLC and $\mathbf{C 6 H}$ appear to be ordered to different degrees, it might be expected that there would be variable contributions from each to the absorption and photoluminescence anisotropy.

In order to understand whether or not the order in the system changes during the polymerisation, we also studied the pre-polymerised liquid mesophase in order to determine whether or not the order of the two monomers C6H and FLLC was similar to that found in the cured films. Molten, unpolymerised films of 5\% FLLC content 
were studied using the 1730 and $2210 \mathrm{~cm}^{-1}$ peaks in a similar fashion to that already described. The films were mounted on a variable temperature hot plate and the temperature dependence of order was measured. Figure 5 shows the dependence of order on reduced temperature for a 5\% FLLC content mixture. For reference, the values obtained for equivalent cured films (taken from Table 2) are also plotted. It is clear that there is agreement between the values for cured and uncured films. This indicates that the polymerisation process does not contribute to any change in order for either C6H or FLLC. The order found in the polymerised films is therefore the same as the order found in the pre-polymerised liquid mesophase.

\section{Experimental}

\section{Differential Scanning Calorimetry (DSC) Measurements}

Measurements were performed on a Perkin Elmer DSC-4. The sample quantities were in the range of $10 \mathrm{mg}$. The heating and cooling rates were $10^{\circ} \mathrm{C}$ per minute, the measurements were carried out under an inert nitrogen atmosphere.

\section{Thin Film Preparation}

Oriented photopolymerised thin films were produced by an oriented rubbing method. A liquid crystal display type glass cell of $10 \mu \mathrm{m}$ thickness was fabricated. The inside walls of the cell were coated with a thin layer (ca. $0.1 \mu \mathrm{m}$ ) of spun cast Nylon 66 which was rubbed along one direction of the film plane in order to induce alignment when the glass cell was filled with liquid crystal material. Long range ordering could not be induced in samples of compound FLLC alone.

Compound FLLC (5\% by weight) was mixed with 1,4-phenylene bis 44 -[6-(acryloyloxy)hexyloxy]benzoate $\}$. 2\% mol. of photoinitiator (CIBA-Irgacure ${ }^{\mathrm{TM}}$ 651) and 0.1 mole percent of thermal inhibitor (4-methoxyphenol) was added.

A glass cell containing the mixture was brought to $130^{\circ} \mathrm{C}$ (nematic phase) and photopolymerised by irradiation for 20 minutes with a low intensity UV fluorescent lamp (4 W) lamp. The cross-linked film thus produced is of very good optical quality and exhibits no sign of phase separation or photodegradation. The same film was used in all subsequent studies.

\section{Raman Spectroscopy Measurements}

Raman spectra were recorded using an Instruments S.A. LABRAM 1B confocal microprobe system operating with a polarised Helium Neon laser at a wavelength of $632.18 \mathrm{~nm}$. The polarisation extinction ratio of the laser was measured to be greater than 500:1. A 50X objective was employed, giving a nominal spot size of $1.2 \mu \mathrm{m}$. The power incident on the sample was $11 \mathrm{~mW}$. Polarisation angle dependent spectra were recorded by rotating the sample in the plane perpendicular to the incident beam. The 
objective was focused before each new spectrum was recorded to ensure completely consistent incident power levels were achieved.

\section{Conclusion}

Molecular order in thin films of a new oriented, cross-linked liquid crystal mixture have been probed in a new manner using Raman anisotropy measurements. These studies show that the two different molecular components in the films are ordered to different degrees. The order parameter for each component has been determined for two Raman peaks and the results are self-consistent.

The dependence of photopolymerisation temperature on order was also probed and it was found that as temperature increases, the degree of order of both molecular components decreases. This has been shown to be independent of the photopolymerisation process itself, the prepolymerised mixture showing similar order characteristics.

The use of Raman spectroscopy has proven to be advantageous in this case where thin film samples are studied. There is also an advantage here due to the greater Raman activity of the carbon-carbon triple bond mode as compared to infrared spectroscopy. Further studies on fabrication and testing of electrically driven devices are in progress with a view to producing a highly polarised light emitting diodes. 


\section{Acknowledgements}

This work was carried out under the EU ESPRIT Long Term Research Programme (LUPO project no. 20038).

\section{References}

1. J. H. Burroughs, D. D. C. Bradley, A. R. Brown, R. N. Marks, K. Mackay, R. H. Friend, P. L. Burns and A. B. Holmes, Nature, 1990, 347, 539.

2. A. Ochse, U. Lemmer, M. Deussen, J. Feldman, A. Greiner, R. F. Mahrt, H. Bässler and E. O. Gobel, Mol. Cryst. Liq. Cryst., 1994, 256, 335.

3. F. Biscarini, O, Greco, A. Lauria, R. Zamboni and C. Taliani, Mol. Cryst. Liq. Cryst., 1996, 290, 203.

4. R. Marks, Proceedings of the NEDO Workshop on Organic Microcavities, Sheffield,1996

5. V. Cimrová, M. Remmers, D. Neher and G. Wegner, Adv. Mater., 1997, in press.

6. G. Lüssem, R. Festag, A. Greiner, C, Schmidt, C. Unterlechner, W. Heitz, J. H. Wendorff, M. Hopmeier and J. Feldmann, Adv. Mater., 1995, 7, 923.

7. D. J. Broer, H. Finkelmann and H. Kondo, Makromol. Chem., 1988, 189, 185.

8 A. P. Davey, R. G. Howard and W. J. Blau, Journal of Materials Chemistry, 1997, 7(3), 417.

9 D. J. Broer, J. Boven, G. N. Mol and G. Challa, Makromol. Chem., 1989, 190, 2255. 


\section{Figure Captions}

Figure 1. Schematic of in situ Photopolymerisation.

Figure 2. Chemical Structure of FLLC

Figure 3. Raman Spectra of the Monomers C6H and FLLC.

Figure 4a. Angular Dependence of Intensity for Peaks associated with FLLC (lines depict fits to Equation 2).

Figure 4b. Angular Dependence of Intensity for Peaks associated with $\mathbf{C 6 H}$ (lines depict fits to Equation 2).

Figure 5. Order parameter for Uncured 5\% FLLC Mixture as a Function of Temperature

Table 1. Values of S for four different Raman modes.

Table 2. $\quad$ Order Parameter Values as a Function of Reduced Temperature. 


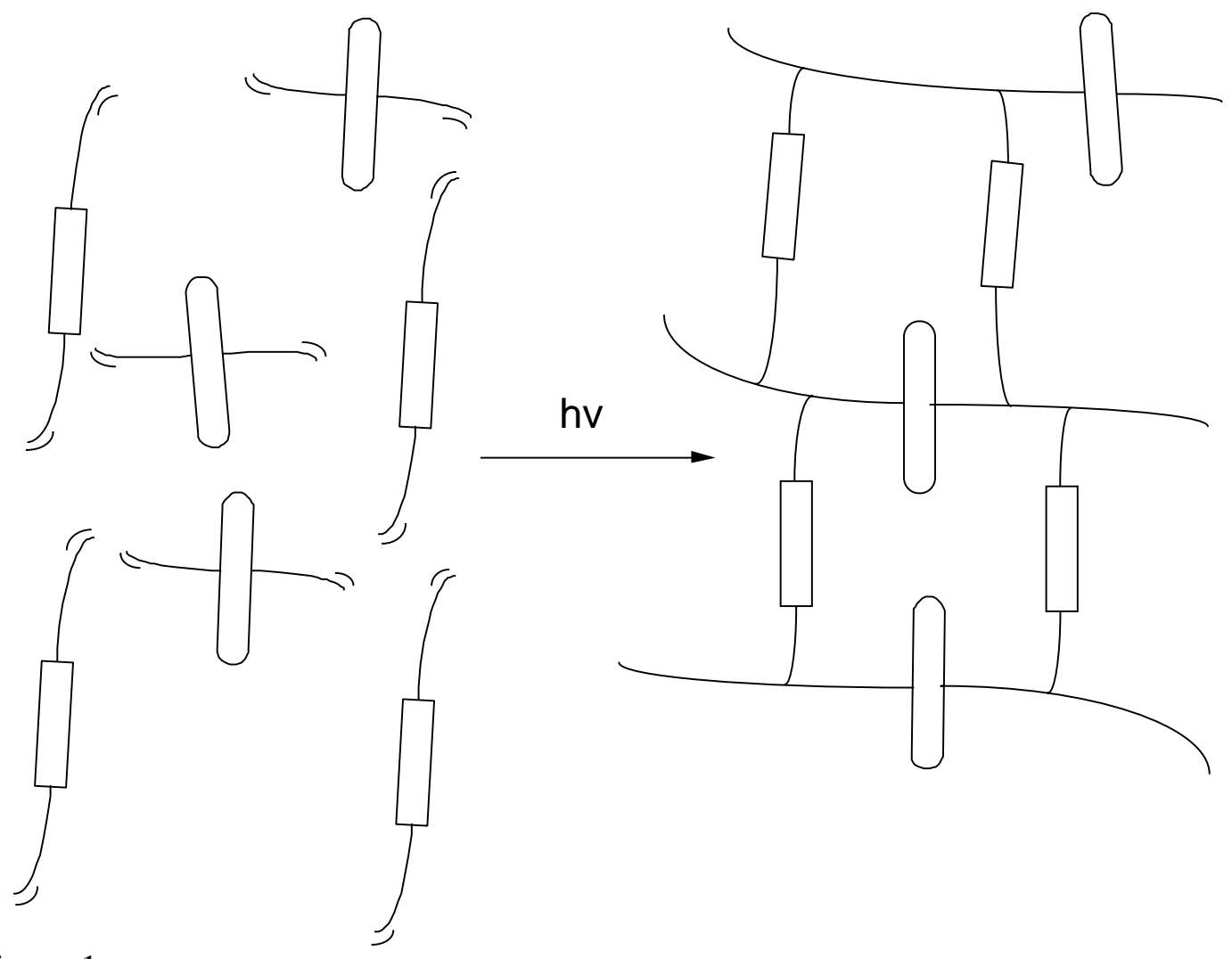

Figure 1. 


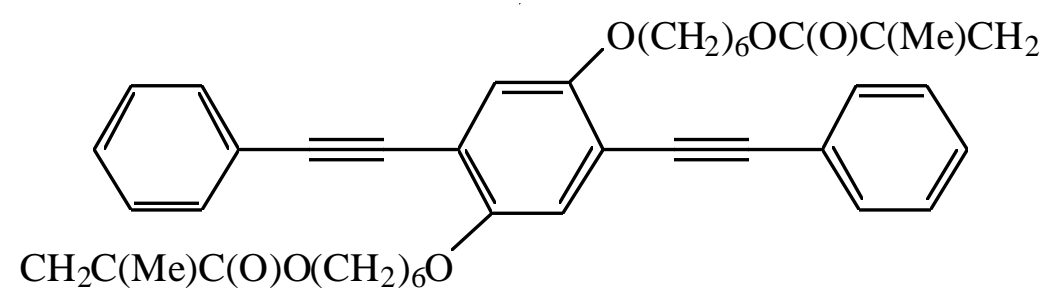

FLLC

Figure 2. 


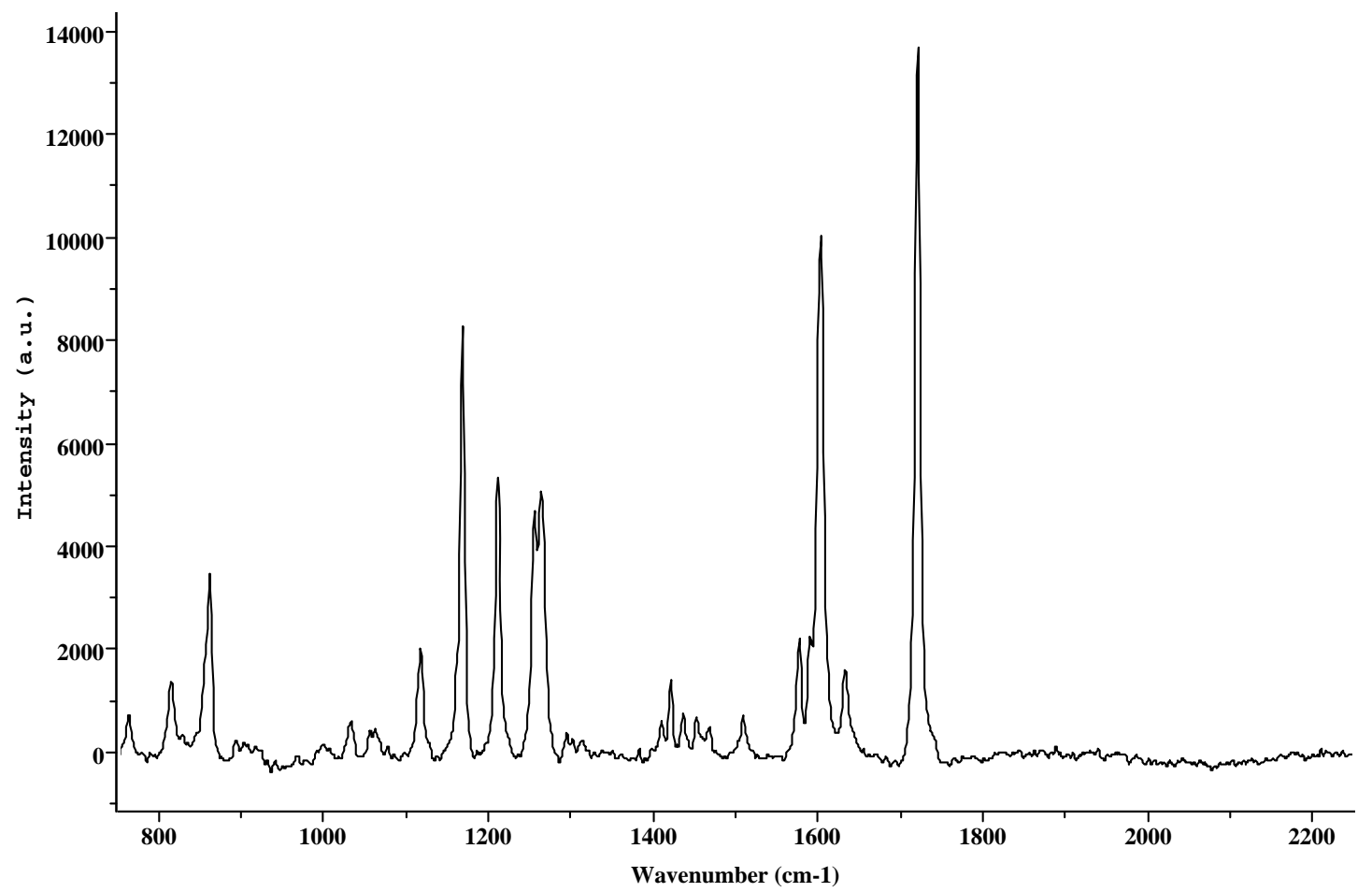

3a: $\mathbf{C 6 H}$

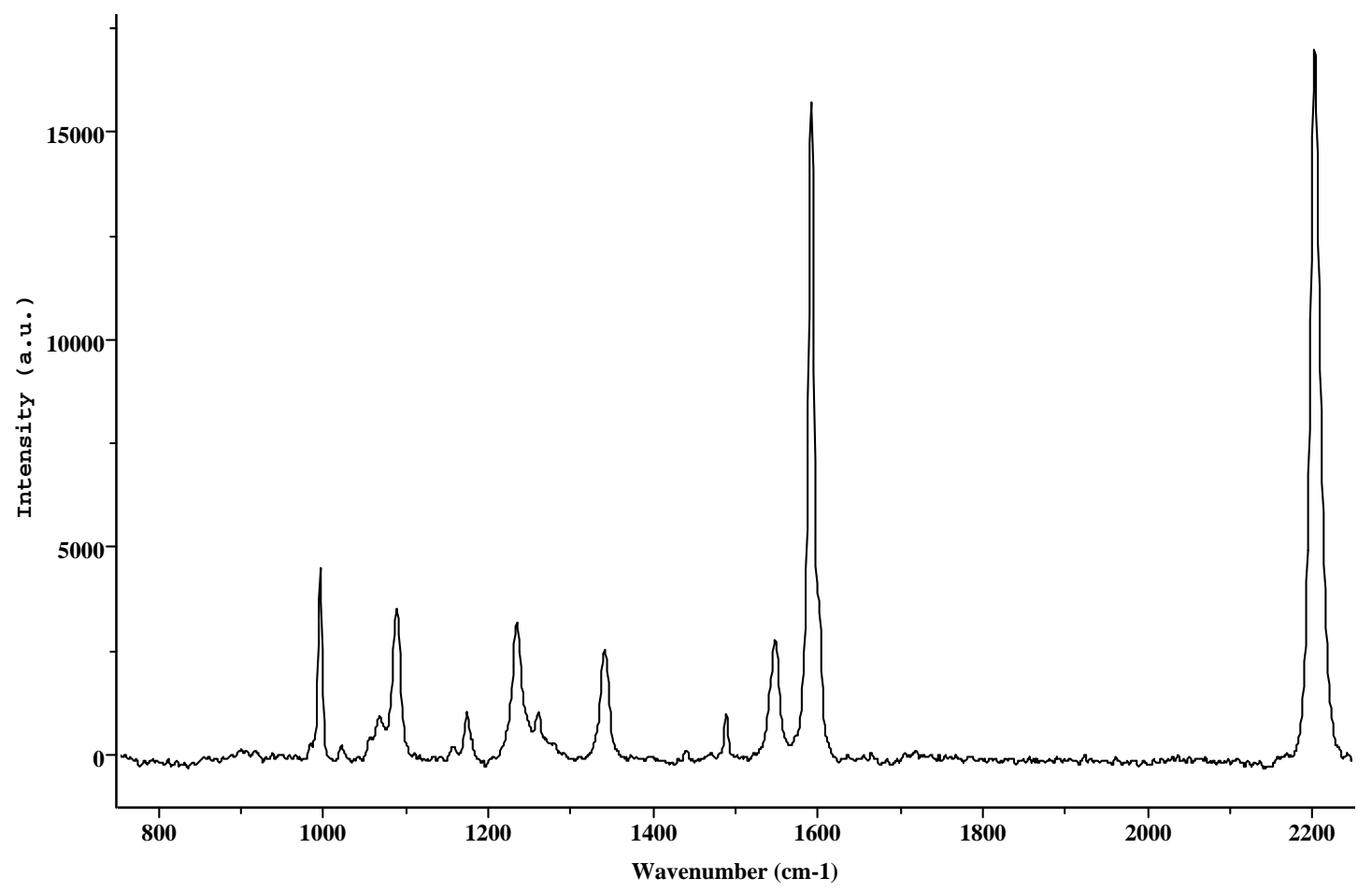

3b: FLLC

Figure 3. 


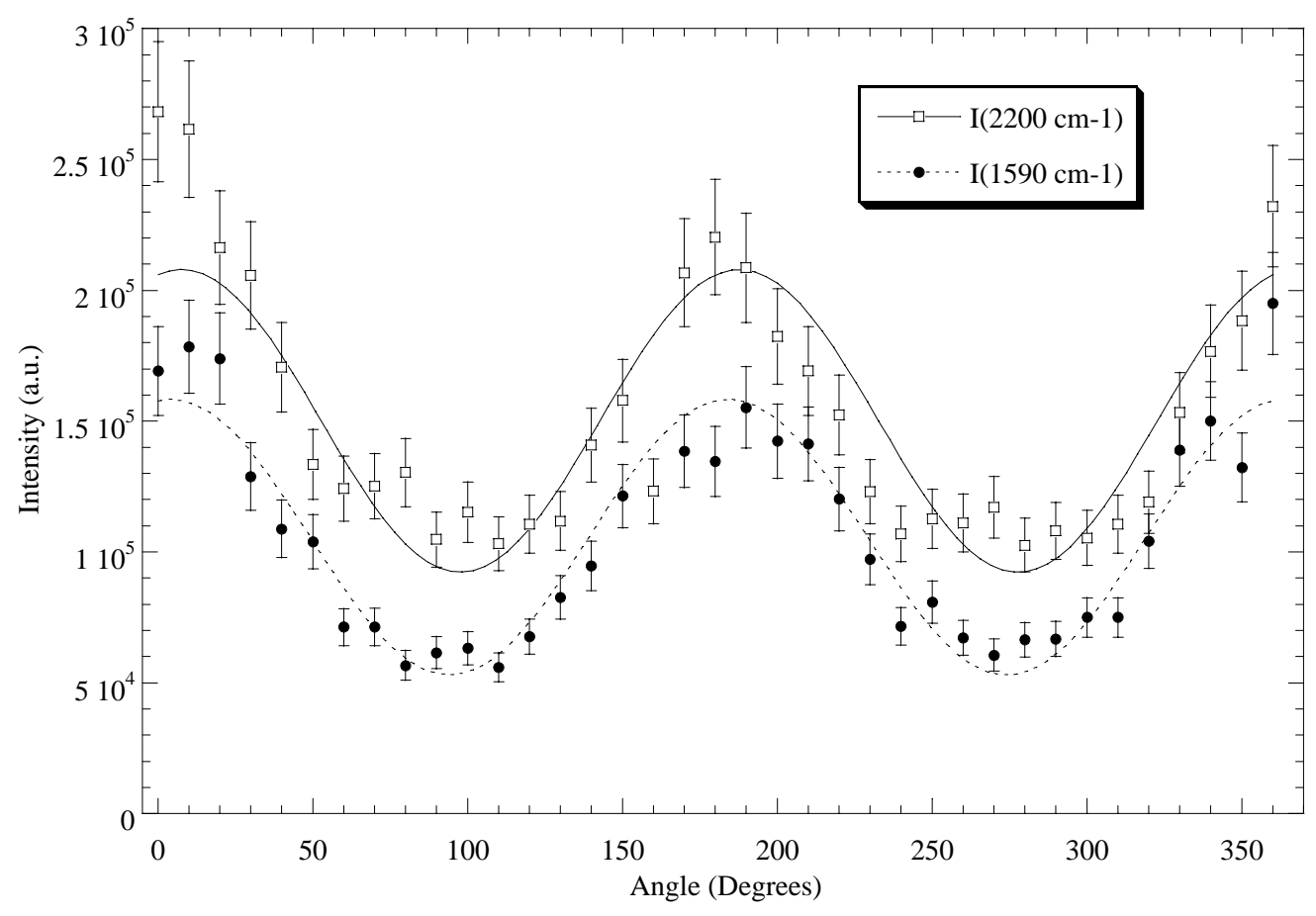

4a

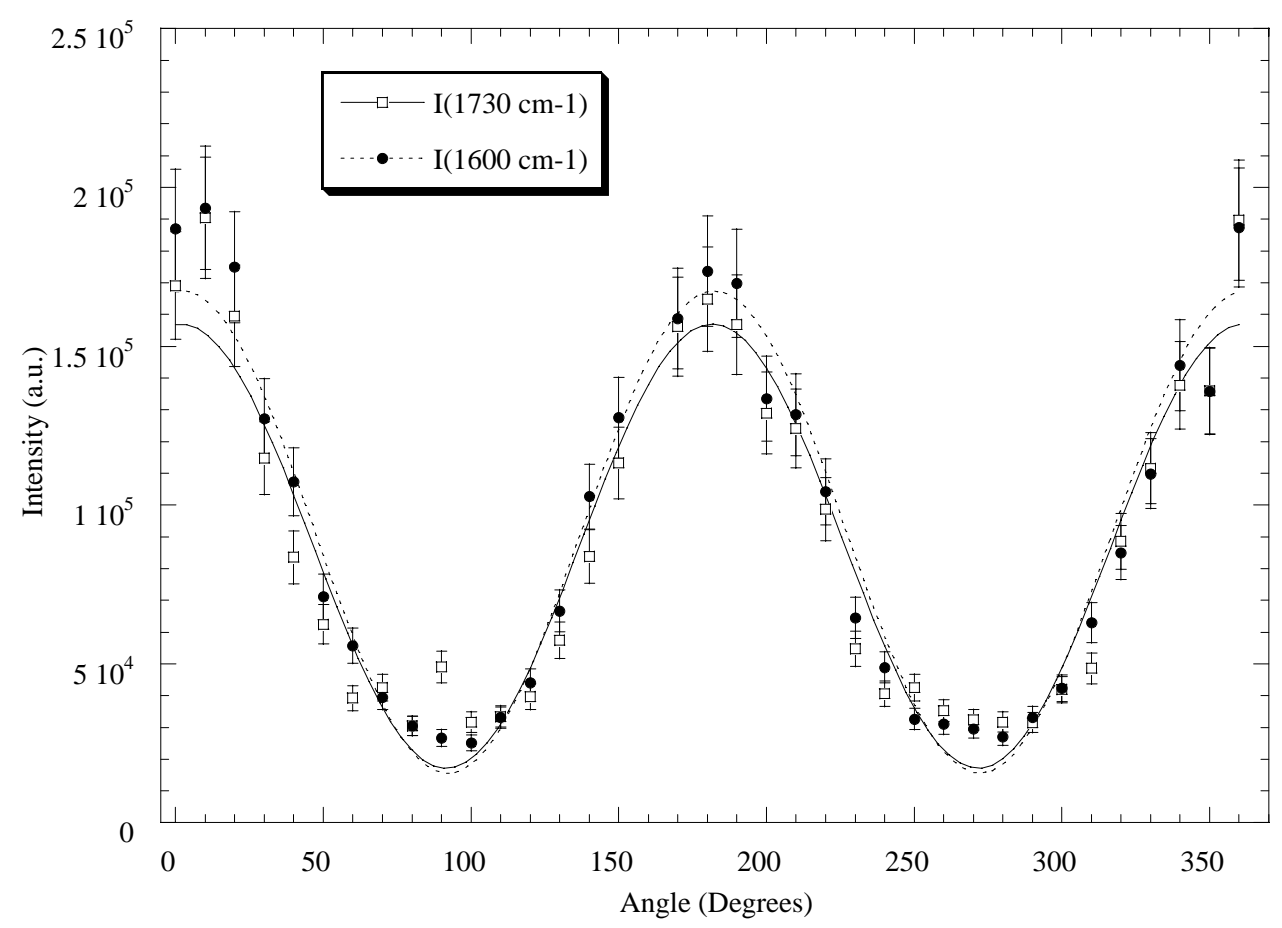

4b

Figure 4. 


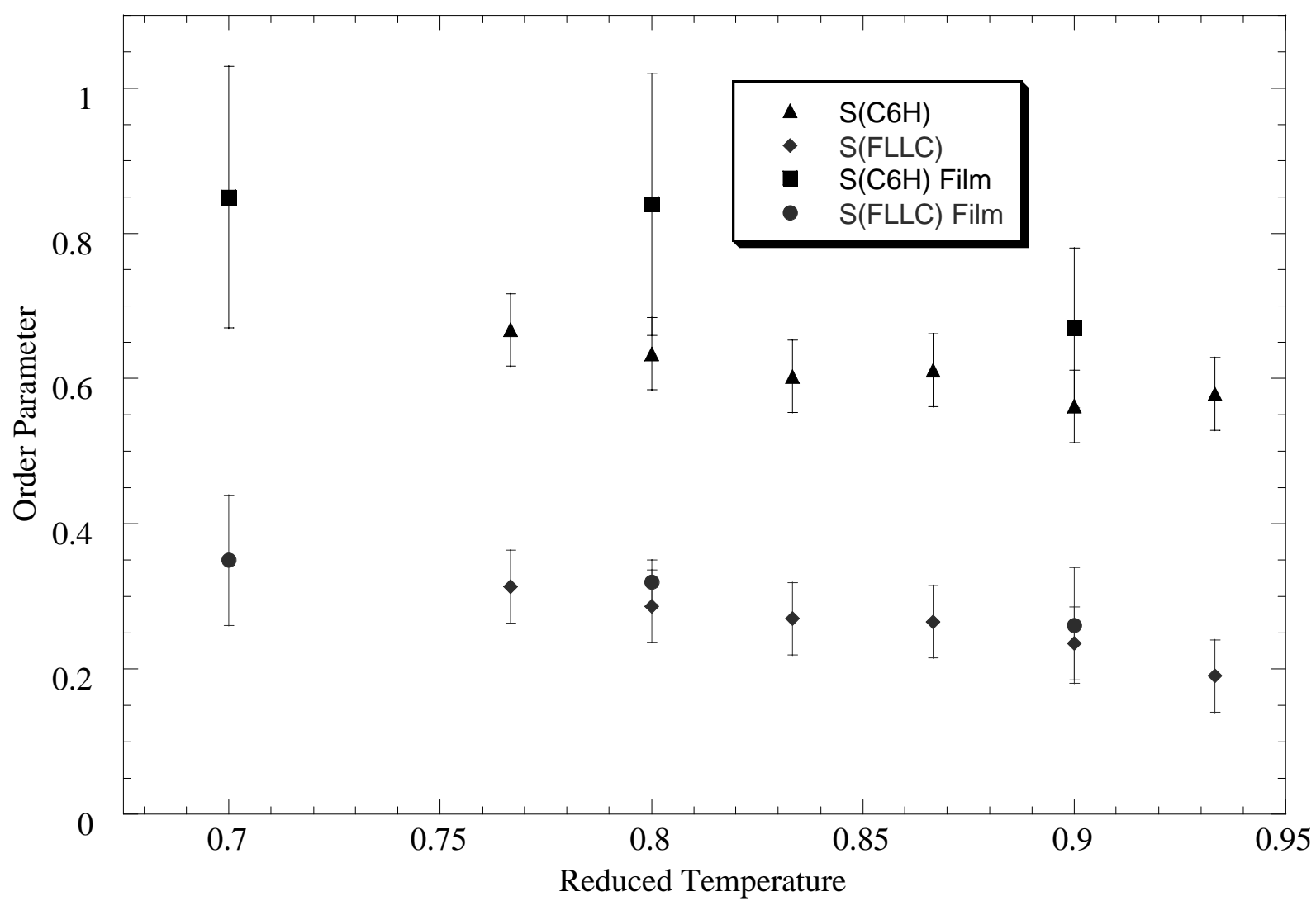

Figure 5. 


\begin{tabular}{|c|c|c|c|c|}
\cline { 2 - 5 } \multicolumn{1}{c|}{} & \multicolumn{2}{c|}{ C6H } & \multicolumn{2}{c|}{ FLLC } \\
\hline Peak & $1600 \mathrm{~cm}^{-1}$ & $1730 \mathrm{~cm}^{-1}$ & $1590 \mathrm{~cm}^{-1}$ & $2200 \mathrm{~cm}^{-1}$ \\
\hline $\mathrm{S}^{*}$ & $0.76 \pm 0.10$ & $0.73 \pm 0.12$ & $0.39 \pm 0.05$ & $0.29 \pm 0.06$ \\
\hline
\end{tabular}

*Errors are derived from fits.

Table 1. 


\begin{tabular}{|c|c|c|}
\hline $\mathrm{S}^{*}$ & $\mathbf{C 6 H}\left(1730 \mathrm{~cm}^{-1}\right)$ & FLLC $\left(2210 \mathrm{~cm}^{-1}\right)$ \\
\hline $\mathrm{T}_{\mathrm{r}}=0.7$ & $0.85 \pm 0.18$ & $0.35 \pm 0.09$ \\
\hline $\mathrm{T}_{\mathrm{r}}=0.8$ & $0.84 \pm 0.18$ & $0.32 \pm 0.03$ \\
\hline $\mathrm{T}_{\mathrm{r}}=0.9$ & $0.67 \pm 0.11$ & $0.26 \pm 0.08$ \\
\hline
\end{tabular}

*Errors are derived from fits.

Table 2. 